\title{
Analysis of Complexity of Unsafe Behavior in Construction Teams and a Multiagent Simulation
}

\author{
Zhen Li $\mathbb{D}^{1}{ }^{1}$ Xiaofei Lv, ${ }^{1}$ Hongming Zhu, ${ }^{1}$ and Zhaohan Sheng ${ }^{2}$ \\ ${ }^{1}$ School of Management, Jiangsu University, Zhenjiang, Jiangsu 212003, China \\ ${ }^{2}$ Computational Experiment Center for Social Science, Nanjing University, Nanjing 210093, China
}

Correspondence should be addressed to Zhen Li; 1000004589@ujs.edu.cn

Received 24 May 2018; Accepted 25 July 2018; Published 23 August 2018

Academic Editor: Shoujun Huang

Copyright (C) 2018 Zhen Li et al. This is an open access article distributed under the Creative Commons Attribution License, which permits unrestricted use, distribution, and reproduction in any medium, provided the original work is properly cited.

\begin{abstract}
The processes in construction are more likely than others to breed unsafe behaviors, and the consequences of these actions can be serious. This paper first reviews the research status on unsafe behavior in construction teams. It then analyzes the complex mechanisms that lead to unsafe behavior and constructs a three-layer structural model based on agent-based modeling (ABM) technology. This modeling deals with complexity and elaborates on key points and potential research ideas in the study of unsafe behavior in construction teams. Using the ABM method, the effects of different incentive strategies on the safe behavior of construction teams under different management scenarios were studied. The results showed that when members have a fair perception of the situation, the effect of the excess performance reward distribution, according to the member's safety awareness level, is better than the average distribution effect. This is the case whether the member's safety behavior level is positively or negatively related to the member's safety awareness level. This study proves the feasibility, validity, and universality of the three-layer structural model. It also reaches certain management conclusions and ideas for further development. The purpose of this paper is to provide a reference for research on the containment and prevention of unsafe behavior in construction teams.
\end{abstract}

\section{Introduction}

According to statistics, about 60,000 construction workers die each year worldwide, which is equivalent to an accident every 9 minutes [1]. In the United States, in 2012, the construction industry reported 806 fatal occupational injuries, accounting for $20.3 \%$ of all fatal occupational injuries in all industries [2]. At present, China is in a period of rapid economic development. The scale of project construction is larger than that in the United States and other developed countries. Therefore, the safety situation in the construction industry is more severe than in developed countries. According to statistics, there were 692 safety accidents in construction projects in China in 2017, including 807 deaths [3].

In the frequency statistics of fatal construction safety accidents published by the U.S. Occupational Safety and Health Administration (OSHA), the top four accidents are directly related to the unsafe behavior of workers. Many theories on causes of accidents also regard the unsafe behavior of workers as a direct cause of safety incidents [4]. Unsafe behavior is defined as any behavior that an employee engages in without regard to safety rules, standards, procedures, instructions, and specific criteria in the system. These actions may adversely affect security systems or endanger the employee or colleagues [5]. Actions of this nature include not wearing a safety helmet, temporarily using an insecure device, ignoring warning signs, and climbing in unsafe locations. Research on unsafe behavior among construction workers is vital to the fundamental prevention and control of safety accidents. This type of study, therefore, has very important theoretical and practical value.

In the process of construction, the project faces enormous challenges such as environmental complexity, technical complexity, and management complexity. As the establishment of key equipment requires the coordination of multiple teams to achieve the optimization of multiple goals such as project progress, cost, and quality, its operational processes are generally based on team cooperation. 
Haslam et al. pointed out that $70 \%$ of construction safety accidents are closely related to the construction team [6]. Therefore, the management and control of unsafe behavior in construction teams are key to safe production and safety management. As a result, it has received extensive attention from academia and industry.

Due to diversity and difference of unsafe behavior in construction teams, there are multiple sources and complexities of motivation. Motivation can be nonlinear and may follow uncertain evolutionary paths. Project conditions such as regulatory cost constraints, incomplete security management systems, and information asymmetry also play a key role in determining behaviors. At an individual level, cognition and decision-making heterogeneity make it difficult to supervise and control the unsafe behavior of construction teams. The practical effect of preventing unsafe behavior of construction teams is thus not ideal. Therefore, analyzing and summarizing the research status of unsafe behavior in the construction team and examining the complex mechanisms that lead to unsafe behavior in construction teams are vital. In addition, seeking research ideas to deal with the complexity of unsafe behavior in construction teams that are realistic is urgently required in the field of engineering management.

To address these issues, this paper first summarizes and analyzes the research status of unsafe behavior in construction teams and divides the existing research into three categories covering three different aspects: subjects' attributes and decision making, subject interaction and environment, and system safety performance. The complexity of unsafe behavior in construction teams and the proposed ABM framework provide the theoretical basis for this study. Secondly, it analyzes the complex and diverse mechanisms governing the subjects' unsafe behavior in the construction team, the interaction between the subjects and the complex environment, and the emergence and dynamic evolution of system performance. On this basis, the method, based on ABM, is applied to the research on unsafe behavior in construction teams to deal with the challenges of their complexity. Based on the research status and the characteristics of the ABM, a multiagent model is constructed. The three-layer structural model of the modular method illustrates the thoughts and technical route of the model in the study of unsafe behavior in construction teams. Finally, the ABM method, based on the three-layer structural model, is applied to study a real case with a realistic background. The evolution mechanism of unsafe behavior in construction teams under different incentive mechanisms proves that the model is feasible, effective, and universal.

\section{Literature Review}

2.1. Individual Cognition and Decision Making in Unsafe Behavior among Construction Teams. Human behavior is the product of cognition. If people's behavior is insecure, it must be a failure in the cognitive process that generates the behavior [7]. From existing research, it is evident that many scholars investigating cognitive psychology, social psychology, organizational psychology, and other theories have analyzed the influencing factors and mechanisms of unsafe behavior in construction teams based on the perspective of individual cognition and decision making. This work has played a positive role in understanding and managing the unsafe behavior of construction workers. We have collated and summarized the relevant literature and conclude that current study on individual behavioral cognition mainly focuses on factors such as psychological factors, cognition and assessment, physiological factors, individual heterogeneous attributes, and historical behavior. The specific classifications and research content are presented in Table 1.

\subsection{Environment and Subject Interaction in Unsafe Behavior} among Construction Teams. With the deepening of research on security incidents, people are gradually realizing that individual safe behavior depends not only on individual cognition but also on other members of the construction team and the operating environment. For example, when insecurity of the operating environment coexists with unsafe behavior of individuals, accidents may occur [29]. The unsafe behavior of the construction team depends on the situation in which it is located; it mainly involves contextual elements such as the objective environment and the realistic basis of the construction team, the interaction mechanism between the internal members of the construction team and different teams, and the interaction mechanism between the construction team and the supervisor. We have collated and summarized the relevant literature, and the specific classifications and research content are shown in Table 2 .

\subsection{System Safety Performance in Unsafe Behavior among} Construction Teams. System safety performance research, as an important part of safety management, helps to clarify the influencing factors and preventive measures of unsafe behavior in construction teams. Firstly, some scholars have analyzed the definition of system safety performance, where the difference is relatively large and can be roughly divided into three categories: one is to directly define the safety performance with the occurrence of safety production accidents and their consequences; the second is to use the actual performance of the company to consider the operational effects of safety work; and the third is to define the safety performance by using accidents and the actual performance of the company [23]. Secondly, some scholars have studied the influencing factors of system safety performance in the construction industry including the degree of influence of the parties involved in the project on safety, project features (such as design and construction complexity), people's insecure behavior, unsafe status of machinery/equipment, onsite environmental insecurity, safety atmosphere and safety awareness, and other factors. In addition, some studies have focused on the measurement methods for construction worker safety performance, which can be broadly divided into two types: lagging indicators and leading indicators $[55,56]$. In particular, one method is the analysis of accident statistics. Safety performance is often reflected by lagging 
TABLE 1: Cognitive and decision-making studies on unsafe behavior in construction teams.

\begin{tabular}{|c|c|c|}
\hline Factors & Subfactors & References \\
\hline \multirow{4}{*}{ Psychological } & Emotion & $\begin{array}{l}\text { People who are in a positive emotional state can better assess the } \\
\text { consequences of their behavior than those who are in a negative emotional state [8]. }\end{array}$ \\
\hline & Attention & $\begin{array}{l}\text { The unsafe behavior of construction workers is mainly due to the } \\
\text { incorrect estimation of potential risks and a lack of attention [9]. }\end{array}$ \\
\hline & \multirow{2}{*}{ Work pressure } & $\begin{array}{l}\text { Hofmann and Stetzer believe that complex and overloaded tasks can } \\
\text { lead to work pressure and thus affect safety behavior [10]. }\end{array}$ \\
\hline & & $\begin{array}{l}\text { The results of the study indicate that work stress is negatively } \\
\text { correlated with safety behavior [11]. }\end{array}$ \\
\hline
\end{tabular}

Satisfaction

The results of the case study showed that the workers in the accident group were dissatisfied with the job compared with the workers in the nonaccident group (control group) [12].

Attitude plays an important role in the behavior of the decision makers [13].

Attitude

Workers perceive risk by collecting various kinds of information, and

Cognition and assessment workers establish safety attitudes based on perceived risks. The workers decided to take action based on the established attitude [14].

Motivational factors (risk/benefit tradeoffs) play an important role in

Motivation the process of violations, and cognitive factors may influence the results of violations. Errors may require the interpretation of individual cognitive processing capabilities [15].

Risk perceptions $\quad$ Risk perception plays a crucial role in eliminating work-related hazards [16].

Fatigue $\quad$ When the miners feel tired, they will be negligent and more vulnerable to unsafe behavior [17].

Occupational injury is related to the age-based curve, with injuries at first increasing with age, then decreasing. The two safety attitude scales

Physiological

Age

Personality

Risk preference

Knowledge

Individual attributes

Work experience

Past behaviors

Historical behavior were related to age, and the elderly are more positive about safety [18].

Young people under the age of 26 have low scores for safety performance, poor safety knowledge, and an aversion to safety management [19].

Studying the relationship between the five dimensions of personality and work-related accidents also found a close correlation between personality traits and worker accident trends [20].

When emphasizing decision-making options for avoiding losses, most people adopt risk-taking strategies [21].

The degree of professional knowledge will directly affect the workers in dealing with professional projects [22].

Employees' safety risk tolerance will be affected by work knowledge and work experience [13].

Work experience affects the safety behavior of workers [23].

Construction workers generally lack objective and rational safety knowledge, and the judgment of the degree of danger is mainly based on personal intuitive experience and past experience [24].

Experienced workers clearly recognize that it is very important to incorporate disaster reduction measures into building technology from the beginning of the project [25].

Goles et al. believe that because of the positive emotional experience brought about by past behaviors, individuals will have a more positive attitude toward such behaviors, which in turn will increase their willingness to implement the behavior again [26].

When experiencing problems, workers often choose a habit as a center of consciousness from experience. Bad habits can lead to injury accidents [27].

Customary unsafe behavior is the "burner" for unsafe production behaviors, and there are seven personal factors that affect habitual violations [28]. indicators such as the number of casualties, accidents, accident rates, and unsafe behavior statistics [57]. The other method is the observation and investigation of the construction site, where safety performance can be reflected by leading indicators such as safety atmosphere and safety awareness [58]. We have collated and summarized the 
TABLE 2: Summary of subject interactions and environment on unsafe behavior in construction teams.

\begin{tabular}{|c|c|c|}
\hline Factors & Subfactors & References \\
\hline \multirow{5}{*}{ Hardware environment } & Construction equipment & $\begin{array}{l}\text { Equipment maintenance has an important effect on the occurrence } \\
\text { and severity of accidents [30]. }\end{array}$ \\
\hline & Weather & $\begin{array}{l}\text { Hot weather conditions will increase the health and safety risks of } \\
\text { construction workers and the possibility of workers suffering from } \\
\text { heat-related diseases [31]. }\end{array}$ \\
\hline & & $\begin{array}{l}\text { The characteristics of miners' workplaces have a major impact on } \\
\text { the occurrence of accidents [32]. }\end{array}$ \\
\hline & Work condition & $\begin{array}{l}\text { Chi et al. studied the impact of unsafe working conditions on unsafe } \\
\text { behavior of workers by analyzing the specific types of accidents and } \\
\text { determining the degree of damage [33]. }\end{array}$ \\
\hline & Technology & $\begin{array}{l}\text { Adeleke et al. showed that there is a significant positive correlation } \\
\text { between technical factors and construction risk management [34]. } \\
\text { In the industrial scene, using RFID technology to locate and monitor } \\
\text { worker's behaviors can help to predict and prevent risks [35]. }\end{array}$ \\
\hline \multirow{13}{*}{ Interaction } & Imitation & $\begin{array}{l}\text { Imitation and learning are the main methods for replicating and } \\
\text { spreading unsafe behaviors. It can lead to or catalyze some } \\
\text { new unsafe behaviors [24]. }\end{array}$ \\
\hline & & $\begin{array}{l}\text { Workers tend to imitate others who appear to have adopted } \\
\text { successful strategies [36]. }\end{array}$ \\
\hline & Social conformity & $\begin{array}{l}\text { Rozin shows how herd characteristics among group } \\
\text { members can affect personal judgment and attitude [37]. }\end{array}$ \\
\hline & & $\begin{array}{l}\text { Safety training plays a crucial role in risk prevention. It can } \\
\text { help builders master the knowledge of construction technology [25]. }\end{array}$ \\
\hline & Safety training & $\begin{array}{c}\text { Training is an indispensable part of a successful scientific and technological } \\
\text { management system. In the design of a technical training program for a } \\
\text { specific position, we should pay more attention to the characteristics of } \\
\text { the staff and the awareness of risk [16]. }\end{array}$ \\
\hline & & Safety training has a positive effect on construction workers' safe behavior [23]. \\
\hline & & $\begin{array}{c}\text { Vredenburgh showed that incentive can effectively reduce } \\
\text { the injury rate in industry [38]. }\end{array}$ \\
\hline & Incentive mechanisms & $\begin{array}{l}\mathrm{Li} \text { et al. explained that the recognition of safety behavior through } \\
\text { bonuses, penalties, awards, advancement, and so on is the most } \\
\text { important factor in motivating us to work safely [39]. }\end{array}$ \\
\hline & Leadership & $\begin{array}{l}\text { Managers' safety management decisions and attitudes have a } \\
\text { significant impact on miners' safety motivation and safety behavior [40]. } \\
\text { Safety leadership measures help to improve safety performance [41]. }\end{array}$ \\
\hline & & $\begin{array}{l}\text { Dov found that the group leader directly influences the employee's } \\
\text { operational behavior through daily task decisions [42]. }\end{array}$ \\
\hline & Teamwork behavior & $\begin{array}{l}\text { The teamwork climate has a significant positive effect on } \\
\text { workgroup members' in-role, extra role, and deference behavior [43]. }\end{array}$ \\
\hline & & Workers' safety behavior is influenced by the perceived teamwork climate [44]. \\
\hline & Supervisory behavior & $\begin{array}{l}\text { The supervisory behavior affects the safe climate of the group and the } \\
\text { safety behavior of the employees [45]. } \\
\text { Khosravi et al. studied the influencing factors of unsafe behavior } \\
\text { from the perspective of safety supervision and discussed the } \\
\text { mechanism of management behavior on unsafe work behavior [46]. }\end{array}$ \\
\hline \multirow{4}{*}{ Soft environment } & Regulations & $\begin{array}{l}\text { Safety regulation has significant effects on safety performance [47]. } \\
\text { Rules and regulations are conducive to construction risk management [34]. }\end{array}$ \\
\hline & Safety management systems & $\begin{array}{l}\text { After using the safety management system, the accident rate of the } \\
\text { project dropped significantly [48]. }\end{array}$ \\
\hline & Organizational structure & $\begin{array}{l}\text { The characteristics of organizational structures in construction } \\
\text { make the effects of social norms on safety more complex [49]. }\end{array}$ \\
\hline & Work schedule & $\begin{array}{l}\text { Work plans have a significant effect on the risk behavior of } \\
\text { construction workers [50]. }\end{array}$ \\
\hline
\end{tabular}


TABLE 2: Continued.

\begin{tabular}{lcc}
\hline Factors & Subfactors & References \\
\hline Safety standards & $\begin{array}{c}\text { National safety standards set out various mechanical safety measures } \\
\text { and procedures to ensure safe work [51]. }\end{array}$ \\
Safety climate & $\begin{array}{c}\text { The safety climate has a significant direct effect on safety behavior [33]. } \\
\text { The safety climate of the construction team is becoming more and } \\
\text { more important in the process of construction safety management, } \\
\text { because the casualty rate of the construction team is very high [52]. } \\
\text { The safety culture has an indirect effect on safety behavior [33]. }\end{array}$ \\
Safety culture & Political & $\begin{array}{c}\text { Political factors help construction companies to reduce risks in } \\
\text { construction activities [34]. }\end{array}$ \\
Eacro environment & $\begin{array}{c}\text { Israelsson and Hansson affirmed a significant relationship between } \\
\text { economic factors and construction risk management [53]. } \\
\text { Insurance companies can minimize losses, and contractors can be } \\
\text { motivated to invest in safety [54]. }\end{array}$ \\
\hline
\end{tabular}

TABLE 3: System safety performance indicators in unsafe behavior in construction teams.

\begin{tabular}{|c|c|}
\hline Author & Indicators \\
\hline Hidley[59] & Leadership qualities, workplace culture, workplace pressure \\
\hline Chen et al. [60] & Work pressure, safety climate, management commitment to safety, supervisor safety perception \\
\hline Skeepers and Mbohwa [61] & Safety culture, safety leadership, attitudes, behaviors of individuals \\
\hline Chinda and Mohamed [62] & Safety attitude, safety behavior, safety culture, safety climate \\
\hline Wehbe et al. [63] & $\begin{array}{c}\text { Individual resilience, supervisor safety perception, safety climate, communication, } \\
\text { organizational factors, work pressure }\end{array}$ \\
\hline Griffin and Neal [64] & Safety knowledge, safety skill, motivation \\
\hline Feng [65] & $\begin{array}{c}\text { Safety investments (investments in basic safety measures and investments in } \\
\text { voluntary safety measures), safety culture }\end{array}$ \\
\hline Dearmond et al. [66] & Safety compliance, safety participation \\
\hline
\end{tabular}

relevant literature on system safety performance indicators, and the specific classification and research contents are shown in Table 3.

In general, the study of unsafe behavior in construction teams is a complex, scenario-dependent problem, because it involves many system elements, heterogeneous entities (e.g., owners, designers, supervisors, contractors, subcontractors, and team members), multiple goals [67] (such as duration, cost, and quality), and so on. To solve this problem, many scholars often analyze it using empirical research, case analysis, and game theory and pay less attention to the complex mechanisms of unsafe behavior in construction teams. In the former method, the construction stage is often based on team cooperation. Team members have different goals, abilities, preferences, and so on, and they have learning and adaptability characteristics, which can lead to diversity of behavior in a multistage interaction process. In addition, the existing research is generally based on a certain time interval of the system, and it seldom considers the dynamic correlation between decision making and unsafe behaviors of the subject at each stage of the system's evolutionary process. It also ignores the interaction between multiple manifestations of insecure behavior in multistage interactions and interactions with other subjects.

\section{Complexity Analysis of Unsafe Behavior in Construction Teams}

Due to the universality of the construction team's unsafe behavior, the diversity of its manifestations, the complexity of the impact mechanisms, and the severity of the consequences, it has gradually attracted the attention of researchers in many fields and has become an important topic for research. This paper asserts that the evolutionary process of unsafe behavior in construction teams can be regarded as a multiagent, multilevel dynamic interaction process, and it may face complex challenges in the process of its research. These challenges are summarized in the following section.

3.1. Multisource and Diversity of Causes and Forms of Unsafe Behavior in Construction Teams. Firstly, there are multisource causes for unsafe behavior in the construction team. There are many factors influencing the unsafe behavior of the subject in the system including the subject's complex relationship and interaction mechanisms with the construction team that are unsafe for the construction team. There is a difference between the mode of action and the effectiveness of the impact, which may have a nonlinear effect 
on the unsafe behavior of the construction team. The subject of unsafe behavior is often the result of a combined effect of many factors, which leads to the multisource characteristics of the unsafe behavior of the subject. For example, Bohm and Harris [68] examined the effect of risk perception factors on workers' safety behavior, but the experimental results showed that there was no significant correlation between the two factors. The reason is that the safety behavior of workers is simultaneously influenced by multiple factors. Another example is the pressure of work progress that can cause a decline in the physiological functioning of workers. At this time, if workers are accompanied by "convenient" motivation, the combination of the two may lead to unsafe behavior by workers.

Secondly, the manifestation of unsafe behaviors of construction team workers are diverse. Members of the construction team are heterogeneous in their external environment, goals, abilities, preferences, and so on. The decision making of subjects is influenced by game choice and active feedback under the conditions of an integrated external environment, self-factors, and other conditions. In addition, the subject's complex psychological and behavioral characteristics can have an important influence on decision making, such as their attitude to trust, concern for reputation, attention to fairness, and risk appetite. Therefore, the subject's behavior and decision making will show diverse characteristics. For different scenarios in the project construction phase, such as schedule control [69], cost control, operating environment conditions, and policy environment, the adaptability of the subject's behavior may lead to different behaviors, resulting in multiple manifestations of unsafe behavior [70], like incorrectly using safety protection supplies, violating the regulations, and paying little attention to protecting oneself and other people.

3.2. Adaptability and Situation Dependencies of Subjects' Unsafe Behavior in Construction Teams. Firstly, the scenarios contained in the construction phase are relatively rich and complex. Many elements in the scenarios may affect unsafe behavior. The influencing factors are mainly the external environment and institutional conditions, the team's own attributes, and the multiagent dynamic interaction process. In addition, there are differences in the acting pathway, impact effectiveness, and transmission mechanisms of various factors on unsafe behavior. For example, the safety behavior of the construction team is affected by differences in factors such as team leader safety awareness, concern for social reputation, and staff quality, knowledge, and skill levels.

Secondly, the generation and development of unsafe behavior also depend on certain objective and realistic foundations. Due to the complex and open operating environment of the project, the numerous stakeholders with heterogeneous characteristics, the interaction of the subject's behavior, and other complicating factors, there may be defects and loopholes in the institutional guarantees and supervision of the team members' behavior. For example, it is difficult for owners to know the safety behavior of the construction team during on-site operations, and there is information asymmetry between the subjects, the standards and norms of safety behavior are subjectively ambiguous to some extent, and they are often difficult to define clearly in contractual terms.

In addition, conflicts, interactions, and games between the construction teams and other stakeholders may also affect safety behavior. The scenario of the construction team is a dynamic collection of multiple stakeholders, numerous distributed resources and information, and other elements. Various elements are intertwined and linked to form a certain level or network structure, which changes continuously with the evolution of the system. Deep indefinite features are also generally present, so the dynamic effects and mutation of scenarios can induce the complexity that leads to insecure behaviors, such as the owner's time limits and cost constraints, the supervision of the unit's safety, and the engineering supply chain member's interactions. In the context of the owner's shortcuts, the construction team may be subject to the owners' administrative orders and controls, so they will emphasize the construction period but ignore safety.

Construction team members are both learning and adaptable in the process of a dynamic interaction. Subjects' decision making is itself an interaction with other individuals and the environment, and through learning, imitation, conformity, and other means, it changes their behavior to adapt to environmental changes. Adaptability creates complexity, and the scene dependence of unsafe behavior in construction teams is a manifestation of their adaptability.

3.3. Multistage Correlation and Dynamic Evolution of Unsafe Behavior in Construction Teams. Firstly, there is a close relationship between the unsafe behavior of the construction team and the main decision making at different stages of the construction project. The unsafe behavior of the construction team may be affected by the results of the previous stage of behavioral decision making and may also affect the behavior of the next stage of the project. For example, under the influence of economic interests or performance goals, the owner's actions to shorten the construction period may cause the construction unit to take on long overtime operations to avoid delays in the construction period, which may cause unsafe behaviors such as fatigue.

Secondly, the unsafe behavior of the construction team is often accompanied by the opportunistic behavior of the system's subjects. For example, in the "Shanghai downstairs incident," various violations by the clients, general contractors, construction workers, supervisors, and management were involved. As long as any one of them seriously implements the rules, security accidents will not happen, but the result has become a tragedy of "mutual cooperation and joint directorship." On the one hand, when there is supervisory dereliction of duties, failure to properly perform or passively perform their own duties and obligations, and other opportunistic behaviors, the behaviors driven by the noted interests may induce construction teams to generate unsafe behavior. On the other hand, when the construction team is motivated by unsafe behavior, it may also try to induce other stakeholders to 
generate opportunistic behaviors (such as "rent seeking" in the supervision process) and then create objective conditions for its unsafe behavior. It can be seen that there may be interdependence, mutual causation, emergence mechanisms, and interactional paths between the opportunistic behavior of stakeholders and the unsafe behavior of construction teams.

In addition, unsafe behaviors of construction teams often have dynamic evolutionary characteristics. In the environment of information asymmetry, inadequate supervision, incomplete safety management systems, and uncertain environments, the construction teams will realize the spread and evolution of security behavior through interaction, coordination, organization, imitation, learning, and conformity, which is based on the team members' own attributes and the external scenarios.

In summary, the above factors lead to multisource, diverse causes and forms of unsafe behavior as well as nonlinearity and uncertainty in the evolutionary path of unsafe behavior in construction teams. In addition, insecure construction environments, inadequate management departments, regulatory cost constraints, incomplete safety management systems, unclear operating standards and specifications, and other objective conditions, as well as inadequate supervision by government regulatory agencies and imperfect insurance systems, have made the supervision and control of unsafe behaviors in construction teams extremely complicated and difficult to understand.

\section{Responding to the Challenges of Complexity: A Three-Layer Structural Model Using Agent- Based Modeling}

4.1. Research on Agent-Based Modeling of Unsafe Behavior in Construction Teams. The complexity of unsafe behavior in construction teams has been widely recognized by researchers, and it poses challenges for us to study the field in more depth. The ABM method, as an important tool in the research of complex systems, has achieved remarkable results in its application in social science-related fields [71]. The ABM method mainly integrates computing technology, complex system theory, and evolution theory. It reproduces the basic scenarios of management activities, microagent behavior characteristics, and correlations through the computer. Based on this arrangement, a research method to reveal management complexity and evolutionary rules is produced. The method can comprehensively consider and carefully describe the heterogeneity, learning, adaptability, behavioral preferences, and interaction and association mechanisms among multiple subjects in the system. To a certain extent, it is difficult to model the unstructured factors in the system, and it is difficult to describe the multilevel dynamic interaction process of multisubjects and then construct complex scenarios that are more in line with actual management activities. At the same time, from the individual microbehavior, we can study the macrolevel features of the construction teams such as selfadaptation and emergence and reveal the microbehavior basis of the system's performance evolution from the mechanism, so that the microbehavior is closely integrated with the macroemergence.

This study introduces the ABM method into the study of unsafe behaviors in construction teams and aims to lay a methodological foundation for the construction of unsafe behavior prevention mechanisms. The study of this issue should follow the spiral of "practice-theory-practice." The research ideas and solutions for the situational adaptation of unsafe behavior in construction teams are shown in Figure 1. During the research process, the points in Figure 1 should be noted.

Based on the background of construction practice, a systematic analysis of unsafe behaviors in construction teams was conducted, and the key scientific management issues were condensed. Using scale analysis, case analysis, empirical research, and in-depth interviews to lay the foundation for building an agent-based modeling to improve accuracy, then based on the engineering practice and empirical evidence, the model assumptions, subject decision rules, and interaction rules were continuously revised to construct a more realistic $\mathrm{ABM}$. In addition, the design of the prevention mechanism of unsafe behavior and theoretical results needed to be repeatedly placed in different scenarios to simulate and test the effects. Then, through in-depth comparison, analysis, and verification of the effectiveness of the implementation for preventing unsafe behavior in construction teams and their long-term evolution, the rational selection and creation of specific strategies were implemented to ensure their robustness and effectiveness. These were analyzed and tested to achieve a reasonable selection and creation of specific strategies to ensure their robustness and effectiveness.

4.2. Three-Layer Structural Model Framework of Unsafe Behavior in Construction Teams. Constructing a multiagent experimental model is an important part of the ABM research on unsafe behavior in construction teams. Based on the ABM research ideas on unsafe behavior in construction teams, this paper proposes a three-layer structural model of ABM. As shown in Figure 2, it consists of three levels, namely, the agent attribute layer, the system agent interaction layer, and the system performance layer. This is an integrated framework that uses $\mathrm{ABM}$ to study the unsafe behavior of construction teams. Each level of subsystems and their elements have their own attributes and input-output relationships that determine the inputs and outputs of the subsystems through interactions. The input and output of the entire system are actually the interaction of various levels of subsystems; therefore, the three-layer structural model constitutes a complete self-evolutionary system.

(1) The system performance layer belongs to the macrolevel of the system and describes the system macro features of the unsafe behaviors in the construction teams that need to be studied, such as system macro performance indicators like safety atmosphere, safety awareness, accident rate, 


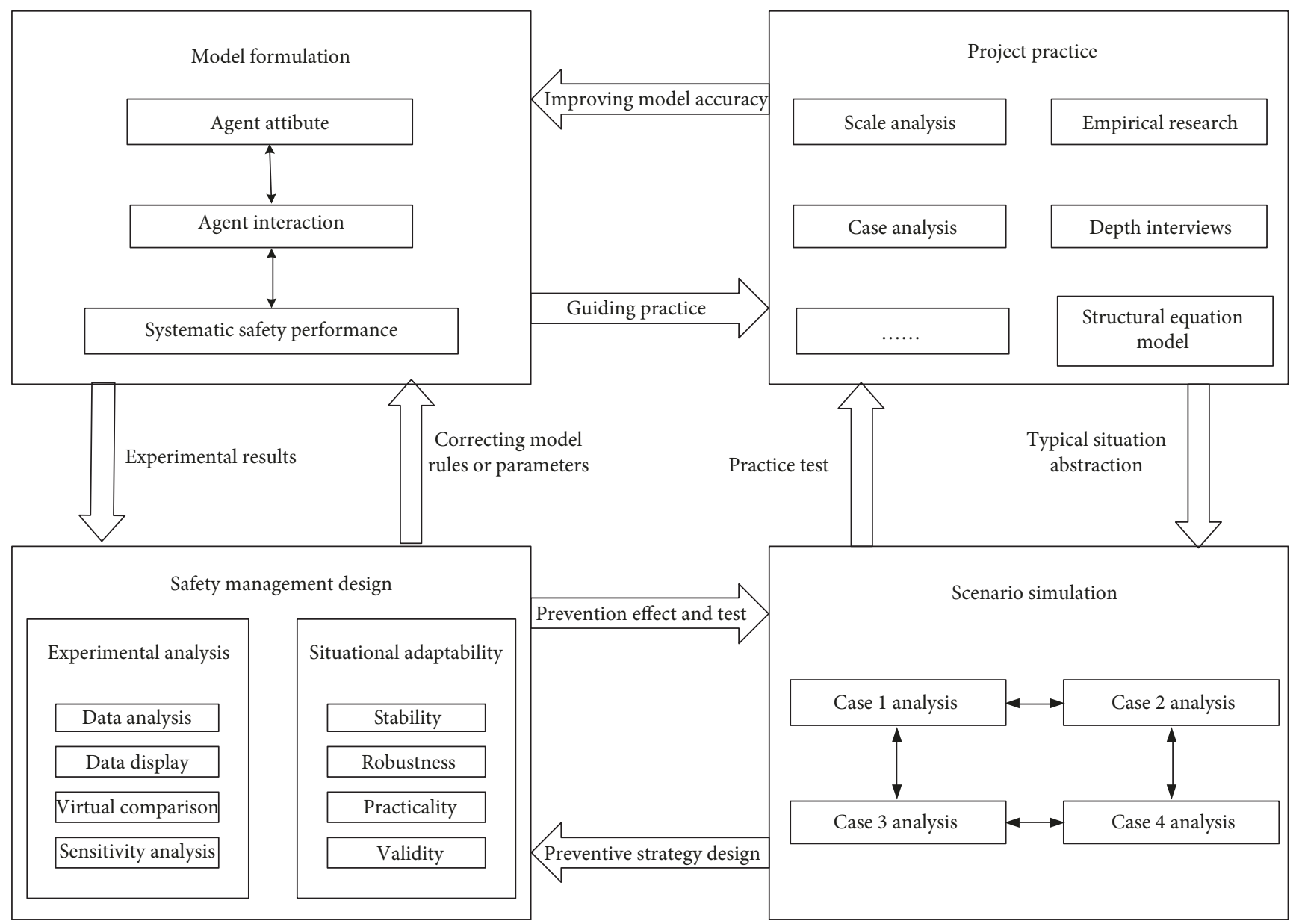

FIGURE 1: Research ideas and design of strategies for prevention of unsafe behavior in construction teams.

number of accidents, and other system macro performance indicators.

(2) The agent interaction layer is mainly used to describe the behavioral characteristics and interaction mechanisms of intelligent agents in the system. It involves the interactive relationship between subject and subject such as learning, imitation, rewards and punishments, and other interactions; it also includes the interaction between subject and environment.

(3) The agent attribute layer is mainly used to describe the basic characteristics of the subject such as individual characteristics, behavioral preferences, and psychological cognition. It is the most basic level in constructing the evolution of artificial social systems and research systems. Based on memory, cognition, behavior, learning, and preference of the subject, this level focuses on the dynamic process of the individual's psychology and behavior in the system, and this layer can abstractly reflect the self-evolutionary mechanism of the intelligent subject.

In the process of system self-evolution, "emergence" is expressed as the output of cognitive decisions formed under the internal interaction of the subject's attribute layer (microlevel), which in turn affects the interactions between the various entities within the interaction layer of the subject and the interaction between the subject and the environment. The results of the interactions serve as input to the system's performance layer and reflect the macrolevel behavioral characteristics and performance in the system performance layer. During the construction process, it exists in the microlevel upward transmission and affects the macrolevel phenomenon. It also has an influence on the impact and feedback of the macrolevel on the microlevel. This is embodied in the setting of system performance indicators, the construction safety climate, and safety culture. Other factors will affect many other factors such as the interaction of the agent within the system, the establishment of safety regulations and penalties, and the layout of the construction environment. The interaction of agents and the situation of the construction environment further feedback and influence the attribute indicators such as cognitive, psychological, and risk perception of each subject. Therefore, in the process of system self-evolution, the three-layer structural model not only has bottom-up "emerging" but also top-down feedback and correction under interaction.

Using the three-layer structural model, we can study the behavior in decision making, interactive games, and the self-organization and self-adaptive evolution of the system 


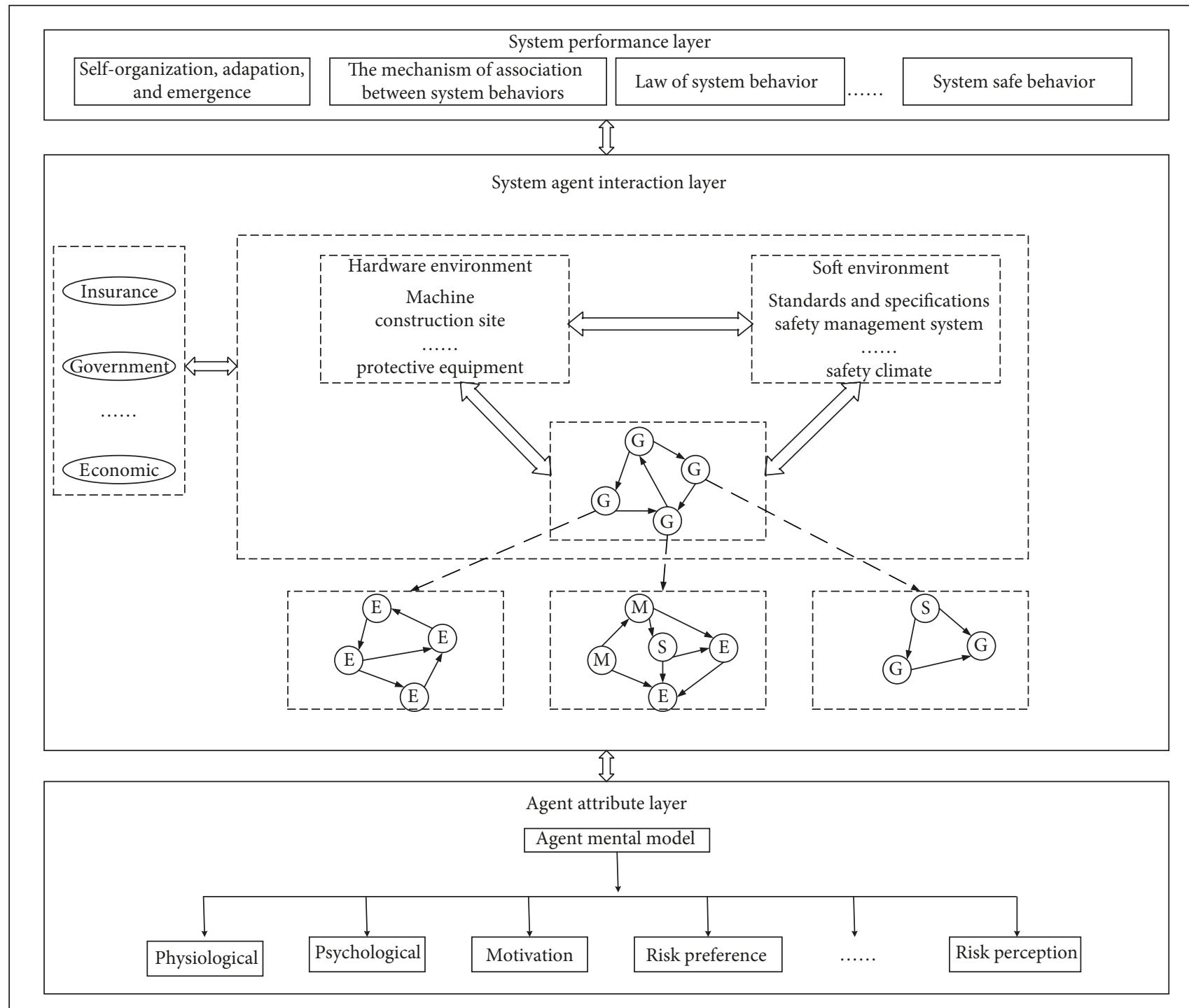

(G) Group (work team)

(E) Employee

(S) Supervisor

Figure 2: Three-layer structural model framework for studying unsafe behavior in construction teams.

in the construction process and then establish the relationship between the subjective microbehavior and the macro emergence of the system. In the process of model building, it is necessary to focus on the modeling of learning and adaptability in the decision-making process of the agent. In the process of multistage construction practice, each agent generally adjusts and optimizes its behavioral decisions based on learning, imitation, trial and error, and so on, and the agent can dynamically adjust its behavior according to its own attributes and external environment.

\section{Case Study}

In order to further verify the universality and effectiveness of the three-layer structural model proposed in
Section 4 and to further elaborate on how to use the ABM method to study the unsafe behavior of construction teams, in this section, we study the evolution of unsafe behavior of construction teams under different incentive scenarios based on the three-layer structural model framework and $\mathrm{ABM}$.

5.1. Experimental Design. A project construction team consists of individuals such as project managers, technicians, professional foremen, quality inspectors, material managers, and safety managers. Team members' safety performance awards consist of basic performance income $\left(a_{i}\right)$ and excessive performance rewards $\left(g_{i}\right)$. Team safety performance is mainly affected by two factors. On the one hand, it is the level of safety behavior $\left(e_{i}\right)$ of members $i$; Vinodkumar 
TABLE 4: Four management scenarios for safety performance incentives.

Management scenario settings

Excessive performance bonus distribution plan

According to the level of safety awareness

Equally distributed

\begin{tabular}{lccc}
\hline $\begin{array}{l}\text { Level of distribution of } \\
\text { member safety behavior }\end{array}$ & Positive correlation with members' safety awareness level & Scenario 1 & Scenario 2 \\
Negative correlation with members' safety awareness level & Scenario 3 & Scenario 4
\end{tabular}

and Bhasi pointed out that safety policies can effectively promote the improvement of safety behavior [72]. During the construction process, workers' degrees of compliance with safety regulations and their degree of compliance with safety standards during the construction process will have a certain impact on their level of safety behavior. On the other hand, looking at the safety awareness level $\left(\lambda_{i}\right)$ of members $i$ in the construction process, Wang and Yuan pointed out that professional knowledge and experience will have an impact on workers' cognition [22]. The level of safety awareness mainly depends on their safety knowledge level, safe work experience, and perception of risk, ability, and so on.

The basic performance income is positively correlated with the team members' safety awareness level, and the basic performance income is the normal distribution $N\left(\mu, \sigma^{2}\right)$. Members differ in basic performance income, which is the size of the variance. The other part is the excess performance reward. The safety supervisors will supervise and evaluate the safety behavior of the construction teams and accept the safety management work. When the safety performance level exceeds the basic safety performance requirement, the team members will receive the excess performance award. Currently, team managers consider two types of safety performance incentives. One is to evenly distribute the excess performance rewards based on team safety performance levels, and the other is to allocate excessive performance rewards based on individual member's safety awareness levels.

This paper considers two types of correlations between $\left(e_{i}\right)$ and $\left(\lambda_{i}\right)$. One is that the level of initial security behavior of members is positively related to their level of security awareness. That is, the higher the level of security awareness of the members, the higher the level of their safety behavior and the higher their awareness of safety norms, systems, and so on. They are also willing to comply and implement it. The other is that the level of initial security behavior of members is negatively related to their level of security awareness. That is, although members have a high degree of awareness of safety norms and systems, they are not willing to comply and implement them in the course of their operations. According to the safety performance incentive plan and the two types of correlations between $\left(e_{i}\right)$ and $\left(\lambda_{i}\right)$, we can construct four kinds of management scenarios accordingly, as shown in Table 4. This paper focuses on four management scenarios to study the impact of safety performance incentives on the evolution of safety behaviors in construction teams.
5.2. Agent-Based Modeling Formulation. Assuming that the team members' safety behavior level is $e_{i}$, the effort cost is $c_{i}=e_{i}^{2} / 2$, the team members' $(i)$ safety awareness level during the construction process is $\left(\lambda_{i}\right)$, then the safety performance of team members is closely related to their individual safety behavior and the level of safety awareness is $\pi_{i}=\lambda_{i} e_{i}$.

The overall safety performance output of the construction team $\Psi$ can be expressed as the sum of individual safety performance, as shown in (1). The owner uses part of the overall safety performance output of the construction team for the members' excessive performance rewards, assuming that the excessive performance reward is $\omega$, then $\omega=\theta \Psi$, where $\theta$ is the proportional coefficient used to reward members for excessive performance in overall safety performance output, $\theta \in(0,1]$.

$$
\Psi=\sum_{i=1}^{n} \lambda_{i} e_{i}
$$

Assuming $\beta_{i}$ is the distribution coefficient of the team members' $(i)$ excessive performance awards, in the process of excessive performance rewards, if the allocation is based on the member's level of safety awareness, then $\beta_{i}=\lambda_{i} / \sum_{j=1}^{n}$ $\lambda_{j}$; if the allocation is based on the average distribution, then $\beta_{i}=1 / n$, which $\sum_{i=1}^{n} P_{i}=1$.

Assuming that team members are risk-neutral, the total income received by each team member based on their safety performance output is the sum of basic performance income and excessive performance incentives, as shown in

$$
v_{i}=\frac{a_{i}+\beta_{i} \theta \Psi-e_{i}^{2}}{2}
$$

Assuming that the team members all have fairness perception. Fairness perception is a key factor in team members forming cooperative relationships, and good partnerships can help improve project management performance. Love learned from interviews that if the contractors feel that in terms of distribution, interaction, and information openness, it is unfair, then this can lead to negative cooperation, and it will in turn affect the contractor's violations and unsafe behavior [73]. During the construction process, construction team members can observe, perceive, and obtain the safety behavior of other team members, and then compare the ratio of their own total revenue $\left(v_{i}\right)$ to the cost of payment $\left(c_{i}\right)$ to the costbenefit ratio of other members. 
TABLE 5: Member security cognition level and initial setting of safety behavior level.

\begin{tabular}{lcccccccccccccccccccc}
\hline$N$ & 1 & 2 & 3 & 4 & 5 & 6 & 7 & 8 & 9 & 10 & 11 & 12 & 13 & 14 & 15 & 16 & 17 & 18 & 19 & 20 \\
\hline$\lambda_{i}$ & 4 & 7 & 9 & 9 & 10 & 11 & 13 & 14 & 14 & 15 & 17 & 19 & 20 & 21 & 22 & 28 & 30 & 33 & 34 & 40 \\
$e_{i}(1)$ & 8 & 9 & 9 & 11 & 12 & 12 & 12 & 12 & 13 & 14 & 17 & 17 & 19 & 26 & 26 & 26 & 29 & 29 & 33 & 40 \\
$e_{i}(2)$ & 40 & 33 & 29 & 29 & 26 & 26 & 26 & 19 & 17 & 17 & 14 & 13 & 12 & 12 & 12 & 12 & 11 & 9 & 9 & 8 \\
\hline
\end{tabular}

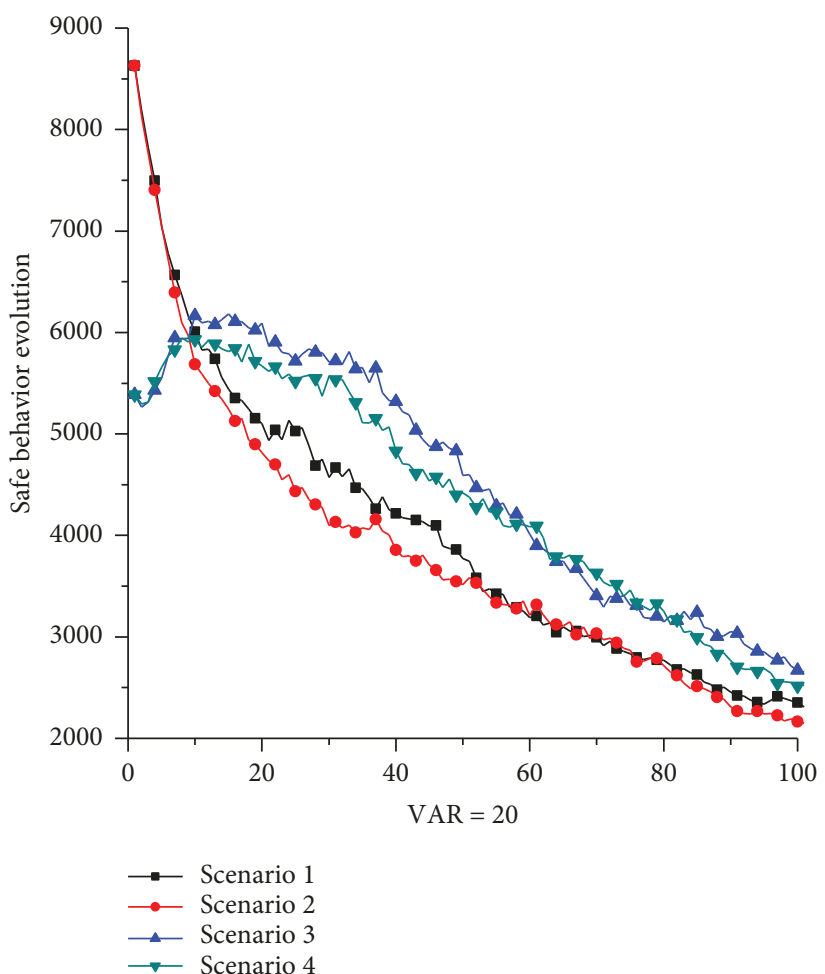

(a)

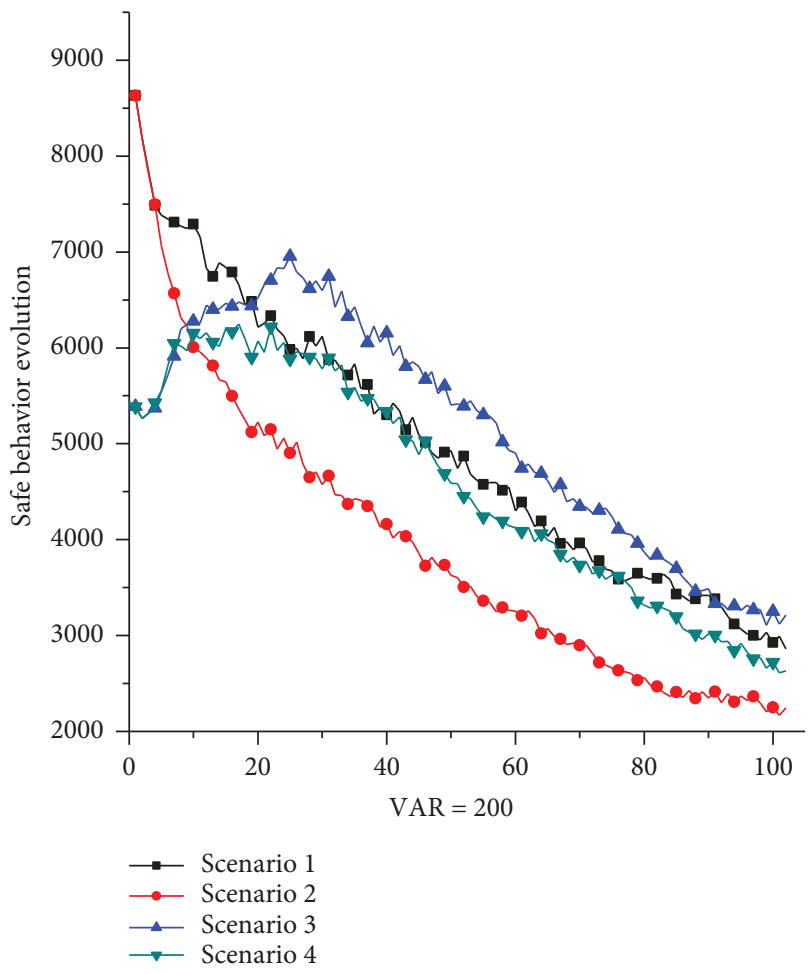

(b)

FIGURE 3: Evolution of safety behavior of construction team members under different variances.

If we adjust the level of safety behavior according to fairness perception, then the specific adjustment rules are shown in

$$
e_{i}(t+1)= \begin{cases}e_{i}(t) \xi_{i}, & \text { if } \frac{v_{i}}{c_{i}}>\frac{\sum_{j \neq i}\left(v_{j} / c_{j}\right)}{n-1}, \\ e_{i}(t), & \text { if } \frac{v_{i}}{c_{i}}=\frac{\sum_{j \neq i}\left(v_{j} / c_{j}\right)}{n-1}, \\ e_{i}(t)\left(1+\mu_{i}\right), & \text { if } \frac{v_{i}}{c_{i}}<\frac{\sum_{j \neq i}\left(v_{j} / c_{j}\right)}{n-1} .\end{cases}
$$

Here, $e_{i}(t+1)$ represents the level of safety behavior of members $(i)$ in the cycle $(t+1)$ : if $\left(v_{i} / c_{i}\right)>\left\{\sum_{j \neq i}\left(v_{j} / c_{j}\right)\right\} /$ $(n-1)$, member $(i)$ will reduce their level of safety behavior; $\xi_{i}$ represents the level of adjustment of the safety behavior, which follows the uniform distribution of $[0.8,1]$, if $\left(v_{i} / c_{i}\right)=\left\{\sum_{j \neq i}\left(v_{j} / c_{j}\right)\right\} /(n-1)$, where member $(i)$ will keep their level of safety behavior in the cycle unchanged, if $\left(v_{i}\right)$ $\left.c_{i}\right)<\left\{\sum_{j \neq i}\left(v_{j} / c_{j}\right)\right\} /(n-1)$, member $(i)$ will increase their safety behavior, the specific adjustment factor is $\mu_{i}$, which obeys the uniform distribution of $[0,0.2]$.

Assuming that the number of members is $20(n=20)$, the members' basic performance income is greater than zero $\left(a_{i}>0\right)$ and determined by normal distribution, then $\mu=250$, considers two scenarios, taking $\{400,40,000\}$, respectively. The safety awareness level $\left(\lambda_{i}\right)$ of construction team members obeys normal distribution $N(20,100)$; the initial settings of team members' safety awareness levels and safety behavior levels are shown in Table 5. Among them, $e_{i}(1)$ indicates that the level of safety behavior of members is positively correlated with the level of safety cognition of members, and $e_{i}(2)$ indicates that the level of safety behavior of members is negatively correlated with the level of safety cognition of members.

5.3. Analysis of Results. First of all, we select the variance of basic performance income $\left(a_{i}\right)$ to be 20 and $200(\sigma=$ $20,200)$, respectively. The evolution trend of the safety behavior of construction team members under the four management scenarios is shown in Figure 3. For the four 
management scenarios, under the action of fair perception of construction teams, the level of safety behavior showed a downward trend. However, based on different allocation schemes for excess performance rewards, evolutionary trends show some regularity and differences.

From Figure 3, we can see the following:

(1) When the level of safety behavior of members is positively or negatively correlated with the level of safety perception of members, the effect of the distribution of excessive performance awards based on the level of member safety awareness is better than the effect of equal distribution.

(2) When the variance of basic performance income is small, although the effect of reward distribution based on members' safety awareness levels is better than the effect of average distribution, the difference is not very obvious. In addition, when the variance of basic performance income increases, the effect of the distribution of excessive performance rewards based on members' safety awareness levels will be greatly enhanced compared with the average distribution effect. It can be seen that the variance of basic performance income will increase the incentive effect of safety behavior to some extent.

(3) The variance of basic performance income is different. The safety behavior of team members in scenario 3 is better than in other scenarios. It can be seen that when the member's safety behavior level is negatively correlated with their safety cognition level, it shows that although the safety norms show good understanding, strong security experience, and high perception of risk, it is not possible to observe and practice safety regulations during the operation process. For such members, the use of rewards based on their level of security awareness will result in better incentive effects.

(4) The safety behavior of team members in scenario 2 is worse than in other scenarios. When the level of safety behavior of members is positively correlated with their level of safety awareness, this type of member has a good perception of safety and is willing to follow safety norms and requirements in construction operations. For such members, if they adopt the method of equal distribution, they will not have a positive incentive effect under the effect of fair perception.

\section{Conclusions}

During the construction phase of the project, team members are more likely to breed unsafe behavior, which may have serious consequences. First of all, this paper reviewed the current research status on unsafe behavior in construction teams from three perspectives: agent cognition and decision making, the interaction among subjects in specific environments, and system safety performance. Secondly, this paper analyzed the multisource factors and diversity of manifestations of unsafe behavior in construction teams, the adaptability and environmental dependence of the agent behavior, and the multistage correlation and dynamic evolutionary characteristics of the unsafe behavior. In addition, ABM was applied to study the unsafe behaviors of construction teams and to deal with the challenges of complexity. Finally, a three-layer structural model based on agent-based modeling was constructed to explain key points and research ideas on unsafe behavior in construction teams.

Using the above research, this paper studied the incentive effects of different incentive strategies for the safety behavior of construction teams under different management scenarios based on the multiagent modeling method and proved the feasibility, effectiveness, and universality of the model. It also offers some management conclusions and inspirations. It aims to provide a reference for research on the containment and prevention of unsafe behaviors in engineering construction teams. The results show that when the level of safety behavior of members is positively or negatively related to the level of safety perception of members, the effect of the distribution of excess performance rewards based on the level of member safety perception is better than the effect of average distribution. Accepting that fair perception exists among team members, when the two are negatively correlated, adopting a reward based on their safety awareness level will achieve better incentive effects, while when the two are positively related, the average incentive is less effective. The variance of basic performance income has an influence on the incentive effect of team members' safety behavior.

The three-layer structural model constructed in this paper has the following limitations. This paper mainly considers the system performance indicators at the macrolevel of the system; however, different risks emerge from a bottom-up system, such as management risk, financial risk, environmental risk, and other risk factors, so at the macrolevel of the system, the design and description of risk indicators and risk assessment methods should be considered. In summary, future research based on the model constructed in this paper should focus on the following: (1) through the identification, feature extraction, and classification of the typical unsafe behavior of members of a construction team, the effects of the heterogeneity of the team members, the complex psychology and behavior preference of the members, the external environment, and the institutional conditions on the unsafe behavior of the members of the team can be further analyzed; (2) the influence of the interaction mechanism (resource allocation, information communication, and other mechanisms) between the members of the construction team and the mechanism of benefit coordination (such as mechanism of reward and punishment and risk sharing) on the evolution of unsafe behavior of team members can be studied; and (3) considering the complex behavior of the members of the construction team, such as psychology, physiology, trust, and imitation learning, the traditional prevention strategy is improved or innovative, and the strategy of preventing unsafe behavior among team members can be further emphasized and built on. 


\section{Data Availability}

The data used to support the findings of this study are available from the corresponding author upon request.

\section{Conflicts of Interest}

The authors declare that there is no conflict of interest regarding the publication of this paper.

\section{Acknowledgments}

This work was supported by the National Natural Science Foundation of China (nos. 71671078, 71501084, and 71390521), Qing-Lan Project of Jiangsu Province, Youth Backbone Teacher Training Project of Jiangsu University, The Key Project of Philosophy and Social Science Research in Colleges and Universities in Jiangsu Province (no. 2018SJZDI052), Senior Personnel Scientific Research Foundation of Jiangsu University (15JDG108), and The National Social Science Fund of China (17AGL010).

\section{References}

[1] Organization I L, "Promotional framework for occupational safety and health," 2006, Report 95 IV (2A), International Labour Organization, https://www.ilo.org.

[2] "Revisions to the 2012 census of fatal occupational injuries (CFOI) counts," 2015, http://www.bls.gov/iif/oshwc/cfoi/cfoi.

[3] "Notice of the Ministry of Housing and Urban-Rural Development on the occurrence of safety accidents in housing municipal engineering in 2017," 2018, Ministry of Housing and Urban-Rural Development of the People's Republic of China (MOHURD), http://www.mohurd.gov.cn.

[4] P. Mitropoulos, G. Cupido, and M. Namboodiri, "Cognitive approach to construction safety: task demand-capability model," Journal of Construction Engineering and Management, vol. 135, no. 9, pp. 881-889, 2009.

[5] I. M. Fam, H. Nikoomaram, and A. Soltanian, "Comparative analysis of creative and classic training methods in health, safety and environment (HSE) participation improvement," Journal of Loss Prevention in the Process Industries, vol. 25, no. 2, pp. 250-253, 2012.

[6] R. A. Haslam, S. A. Hide, A. G. F. Gibb et al., "Contributing factors in construction accidents," Applied Ergonomics, vol. 36, no. 4, pp. 401-415, 2005.

[7] M. Zhang and D. Fang, "Cognitive causes of construction worker's unsafe behaviors and management measures," China Civil Engineering Journal, vol. 45, pp. 297-305, 2012.

[8] J. P. Forgas, G. H. Bower, and S. E. Krantz, "The influence of mood on perceptions of social interactions," Journal of Experimental Social Psychology, vol. 20, no. 6, pp. 497-513, 1984.

[9] D. Wang, J. Chen, D. Zhao, F. Dai, C. Zheng, and X. Wu, "Monitoring workers' attention and vigilance in construction activities through a wireless and wearable electroencephalography system," Automation in Construction, vol. 82, pp. 122-137, 2017.

[10] D. A. Hofmann and A. Stetzer, "The role of safety climate and communication in accident interpretation: implications for learning from negative events," Academy of Management Journal, vol. 41, no. 6, pp. 644-657, 1998.

[11] K. Amponsah-Tawaih and M. A. Adu, "Work pressure and safety behaviors among health workers in Ghana: the moderating role of management commitment to safety," Safety and Health at Work, vol. 7, no. 4, pp. 340-346, 2016.

[12] P. S. Paul and J. Maiti, "The role of behavioral factors on safety management in underground mines," Safety Science, vol. 45, no. 4, pp. 449-471, 2007.

[13] J. Wang, P. X. W. Zou, and P. P. Li, "Critical factors and paths influencing construction workers' safety risk tolerances," Accident Analysis \& Prevention, vol. 93, pp. 267-279, 2016.

[14] Y. H. Shin, S. M. Lee, and I. Yun, "Effect of tilted angle in trench structure on phase change memory operation using TCAD simulation," ECS Transactions, vol. 60, no. 1, pp. 995-999, 2014.

[15] J. Reason, A. Manstead, S. Stradling, J. Baxter, and K. Campbell, "Errors and violations on the roads: a real distinction?," Ergonomics, vol. 33, no. 10-11, pp. 1315-1332, 1990.

[16] G. E. Gürcanl, S. Baradan, and M. Uzun, "Risk perception of construction equipment operators on construction sites of Turkey," International Journal of Industrial Ergonomics, vol. 46, pp. 59-68, 2015.

[17] H. Li, J. Xue, and Y. Yang, "ERP experiment research on impact of fatigue on unsafe behaviors of miners," Journal of Xi'an University of Science and Technology, vol. 3, pp. 376380, 2015.

[18] O. L. Siu, D. R. Phillips, and T. W. Leung, "Age differences in safety attitudes and safety performance in Hong Kong construction workers," Journal of Safety Research, vol. 34, no. 2, pp. 199-205, 2003.

[19] N. Dedobbeleer and P. German, "Safety practices in construction industry," Journal of Occupational Medicine, vol. 29, no. 11, pp. 863-868, 1987.

[20] S. Clarke, "Contrasting perceptual, attitudinal and dispositional approaches to accident involvement in the workplace," Safety Science, vol. 44, no. 6, pp. 537-550, 2006.

[21] K. J. Ko and Z. Huang, "Time-inconsistent risk preferences in a laboratory experiment," Review of Quantitative Finance and Accounting, vol. 39, no. 4, pp. 471-484, 2012.

[22] J. Wang and H. Yuan, "Factors affecting contractors' risk attitudes in construction projects: case study from China," International Journal of Project Management, vol. 29, no. 2, pp. 209-219, 2011.

[23] E. Sawacha, S. Naoum, and D. Fong, "Factors affecting safety performance on construction sites," International Journal of Project Management, vol. 17, no. 5, pp. 309-315, 1999.

[24] Y. Han, Q. Mei, S. X. Liu, and Y. Sun, "Formation of construction workers' habitual unsafe behaviors and factors influencing it," China Safety Science Journal, vol. 25, pp. 29-35, 2015.

[25] K. Chmutina and J. Rose, "Building resilience: knowledge, experience and perceptions among informal construction stakeholders," International Journal of Disaster Risk Reduction, vol. 28, pp. 158-164, 2018.

[26] T. Goles, B. Jayatilaka, B. George et al., "Softlifting: exploring determinants of attitude," Journal of Business Ethics, vol. 77, no. 4, pp. 481-499, 2008.

[27] G. Fu, B. Lu, X. Chen et al., "Behavior based model for organizational safety management," China Safety Science Journal, vol. 15, pp. 21-27, 2005. 
[28] N.-W. Li, M.-H. Xu, and L.-X. Niu, "Study on factors influencing miners' habitual violation behavior based on ISM and AHP," China Safety Science Journal, no. 8, pp. 22-28, 2012.

[29] R. M. Choudhry and D. Fang, "Why operatives engage in unsafe work behavior: investigating factors on construction sites," Safety Science, vol. 46, no. 4, pp. 566-584, 2008.

[30] E. Kazan and M. A. Usmen, "Worker safety and injury severity analysis of earthmoving equipment accidents," Journal of Safety Research, vol. 65, pp. 73-81, 2018.

[31] M. N. Alshebani and G. Wedawatta, "Making the construction industry resilient to extreme weather: lessons from construction in hot weather conditions," Procedia Economics and Finance, vol. 18, pp. 635-642, 2014.

[32] J. Maiti and A. Bhattacherjee, "Evaluation of risk of occupational injuries among underground coal mine workers through multinomial Logit analysis," Journal of Safety Research, vol. 30, no. 2, pp. 93-101, 1999.

[33] S. Chi, S. Han, and D. Y. Kim, "Relationship between unsafe working conditions and workers' behavior and impact of working conditions on injury severity in U.S. construction industry," Journal of Construction Engineering and Management, vol. 139, no. 7, pp. 826-838, 2013.

[34] A. Q. Adeleke, A. Y. Bahaudin, A. M. Kamaruddeen et al., "The influence of organizational external factors on construction risk management among Nigerian construction companies," Safety and Health at Work, vol. 9, no. 1, pp. 115-124, 2018.

[35] J. Chai, C. Wu, C. Zhao et al., "Reference tag supported RFID tracking using robust support vector regression and Kalman filter," Advanced Engineering Informatics, vol. 32, pp. 1-10, 2017.

[36] M. L. Ndeffo Mbah, J. Liu, C. T. Bauch et al., "The impact of imitation on vaccination behavior in social contact networks," PLoS Computational Biology, vol. 8, no. 4, article e1002469, 2012.

[37] P. Rozin, "Social psychology and science: some lessons from Solomon Asch," Personality and Social Psychology Review, vol. 5, no. 1, pp. 2-14, 2001.

[38] A. G. Vredenburgh, "Organizational safety: which management practices are most effective in reducing employee injury rates?," Journal of Safety Research, vol. 33, no. 2, pp. 259-276, 2002.

[39] R. Y. M. Li and S. W. Poon, "Construction safety motivations in Hong Kong: a psychological perspective," in Construction Safety and Waste Management, pp. 111-121, Springer, Cham, 2015.

[40] L. X. Niu, L. I. Nai-Wen, and Q. S. Jiang, "SEM of safety leadership, safety motivation and safety behavior," China Safety Science Journal, vol. 25, no. 4, pp. 23-29, 2015.

[41] C. Wu, N. Li, and D. Fang, "Leadership improvement and its impact on workplace safety in construction projects: a conceptual model and action research," International Journal of Project Management, vol. 35, no. 8, pp. 1495-1511, 2017.

[42] Z. Dov, "Safety climate and beyond: a multi-level multiclimate framework," Safety Science, vol. 46, no. 3, pp. 376387, 2008.

[43] A. M. Anvuur and M. M. Kumaraswamy, "Effects of teamwork climate on cooperation in crossfunctional temporary multiorganization workgroups," Journal of Construction Engineering and Management, vol. 142, no. 1, article 04015054, 2016.
[44] B. Choi, S. Ahn, and S. H. Lee, "Role of social norms and social identifications in safety behavior of construction workers. I: theoretical model of safety behavior under social influence," Journal of Construction Engineering and Management, vol. 143, no. 5, article 04016124, 2017.

[45] D. Fang, C. Wu, and H. Wu, "Impact of the supervisor on worker safety behavior in construction projects," Journal of Management in Engineering, vol. 31, no. 6, article 04015001, 2015.

[46] Y. Khosravi, H. Asilian-Mahabadi, E. Hajizadeh, N. Hassanzadeh-Rangi, H. Bastani, and A. H. Behzadan, "Factors influencing unsafe behaviors and accidents on construction sites: a review," International Journal of Occupational Safety and Ergonomics, vol. 20, no. 1, pp. 111-125, 2015.

[47] H. L. Chang and C. C. Yeh, "Factors affecting the safety performance of bus companies-the experience of Taiwan bus deregulation," Safety Science, vol. 43, no. 5-6, pp. 323-344, 2005.

[48] N. S. N. Yiu, N. N. Sze, and D. W. M. Chan, "Implementation of safety management systems in Hong Kong construction industry - a safety practitioner's perspective," Journal of Safety Research, vol. 64, pp. 1-9, 2018.

[49] H. C. Lingard, T. Cooke, and N. Blismas, "Safety climate in conditions of construction subcontracting: a multi-level analysis," Construction Management and Economics, vol. 28, no. 8, pp. 813-825, 2010.

[50] S. S. Man, A. H. S. Chan, and H. M. Wong, "Risk-taking behaviors of Hong Kong construction workers - a thematic study," Safety Science, vol. 98, pp. 25-36, 2017.

[51] A. A. Raheem and J. W. Hinze, "Disparity between construction safety standards: a global analysis," Safety Science, vol. 70, pp. 276-287, 2014.

[52] Q. Li, C. Ji, J. Yuan, and R. Han, “Developing dimensions and key indicators for the safety climate within China's construction teams: a questionnaire survey on construction sites in Nanjing," Safety Science, vol. 93, pp. 266-276, 2017.

[53] N. Israelsson and B. Hansson, "Factors influencing flexibility in buildings," Structural Survey, vol. 27, pp. 148-161, 2009.

[54] R. Awwad, C. A. Shdid, and R. Tayeh, "Agent-based model for simulating construction safety climate in a market environment," Journal of Computing in Civil Engineering, vol. 31, no. 1, article 05016003, 2017.

[55] R. I. N. Director, WP 53- Thinking about process safety indicators, Australian National University, 2013.

[56] K. Øien, I. B. Utne, and I. A. Herrera, "Building safety indicators: part 1 - theoretical foundation," Safety Science, vol. 49, no. 2, pp. 148-161, 2011.

[57] S. T. Ng, K. P. Cheng, and R. M. Skitmore, "A framework for evaluating the safety performance of construction contractors," Building and Environment, vol. 40, no. 10, pp. 1347-1355, 2005.

[58] J. Hinze, S. Thurman, and A. Wehle, "Leading indicators of construction safety performance," Safety Science, vol. 51, no. 1, pp. 23-28, 2013.

[59] J. H. Hidley, "Critical success factors for behavior-based safety," Professional Safety, vol. 43, pp. 30-34, 1998.

[60] Y. Chen, B. Mccabe, and D. Hyatt, "Impact of individual resilience and safety climate on safety performance and psychological stress of construction workers: a case study of the Ontario construction industry," Journal of Safety Research, vol. 61, pp. 167-176, 2017. 
[61] N. C. Skeepers and C. Mbohwa, "A study on the leadership behaviour, safety leadership and safety performance in the construction industry in South Africa," Procedia Manufacturing, vol. 4, pp. 10-16, 2015.

[62] T. Chinda and S. Mohamed, "Structural equation model of construction safety culture," Engineering Construction and Architectural Management, vol. 15, no. 2, pp. 114-131, 2008.

[63] F. Wehbe, M. A. Hattab, and F. Hamzeh, "Exploring associations between resilience and construction safety performance in safety networks," Safety Science, vol. 82, pp. 338-351, 2016.

[64] M. A. Griffin and A. Neal, "Perceptions of safety at work: a framework for linking safety climate to safety performance, knowledge, and motivation," Journal of Occupational Health Psychology, vol. 5, no. 3, pp. 347-358, 2000.

[65] Y. Feng, "Effect of safety investments on safety performance of building projects," Safety Science, vol. 59, pp. 28-45, 2013.

[66] S. Dearmond, A. E. Smith, C. L. Wilson, P. Y. Chen, and K. P. Cigularov, "Individual safety performance in the construction industry: development and validation of two short scales," Accident; Analysis and Prevention, vol. 43, no. 3, pp. 948954, 2011.

[67] C. Zhao, C. Wu, J. Chai et al., "Decomposition-based multiobjective firefly algorithm for RFID network planning with uncertainty," Applied Soft Computing, vol. 55, pp. 549-564, 2017.

[68] J. Bohm and D. Harris, "Risk perception and risk-taking behavior of construction site dumper drivers," International Journal of Occupational Safety and Ergonomics, vol. 16, no. 1, pp. 55-67, 2010.

[69] S. Tao, C. Wu, Z. Sheng, and X. Wang, "Space-time repetitive project scheduling considering location and congestion," Journal of Computing in Civil Engineering, vol. 32, no. 3, article 04018017, 2018.

[70] A. Suraji, A. R. Duff, and S. J. Peckitt, "Development of causal model of construction accident causation," Journal of Construction Engineering and Management, vol. 127, no. 4, pp. 337-344, 2001.

[71] S. Tisue and U. Wilensky, "NetLogo: a simple environment for modeling complexity," in International Conference on Complex Systems, Boston, MA, USA, May 2004.

[72] M. N. Vinodkumar and M. Bhasi, "A study on the impact of management system certification on safety management," Safety Science, vol. 49, no. 3, pp. 498-507, 2011.

[73] P. E. D. Love, P. R. Davis, R. Chevis, and D. J. Edwards, "Risk/ reward compensation model for civil engineering infrastructure alliance projects," Journal of Construction Engineering and Management, vol. 137, no. 2, pp. 127-136, 2011. 


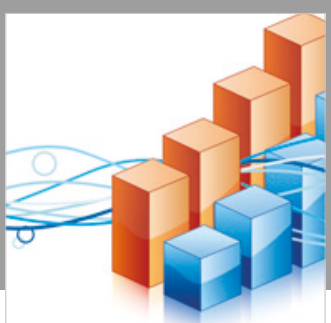

Advances in

Operations Research

\section{-n-m}
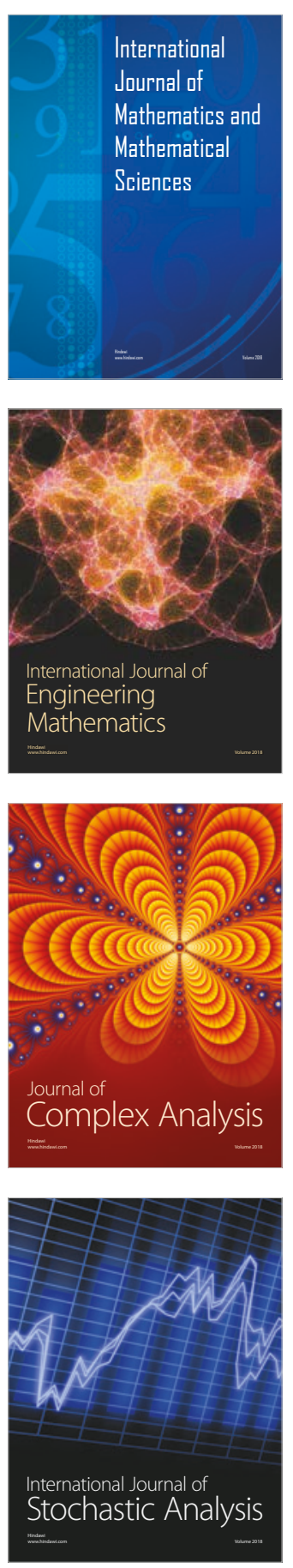
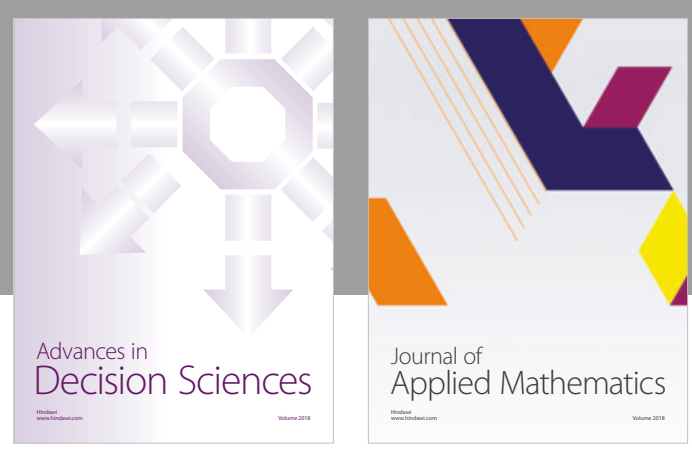

Journal of

Applied Mathematics
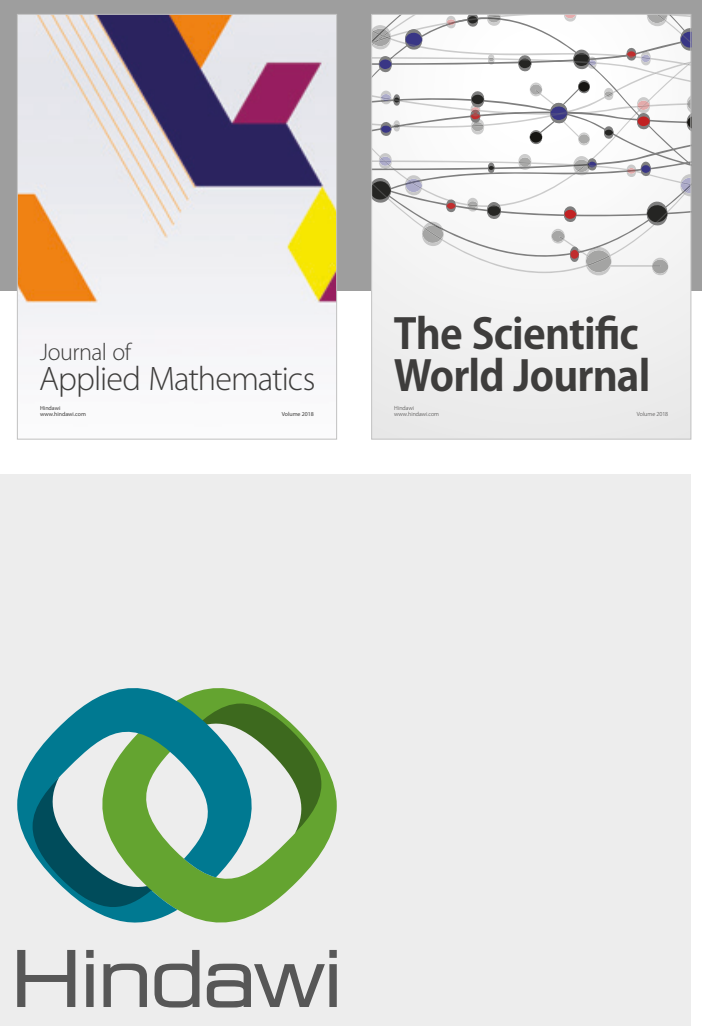

Submit your manuscripts at

www.hindawi.com

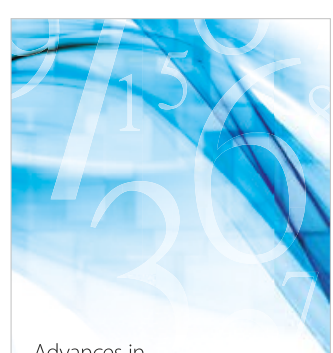

Advances in
Numerical Analysis
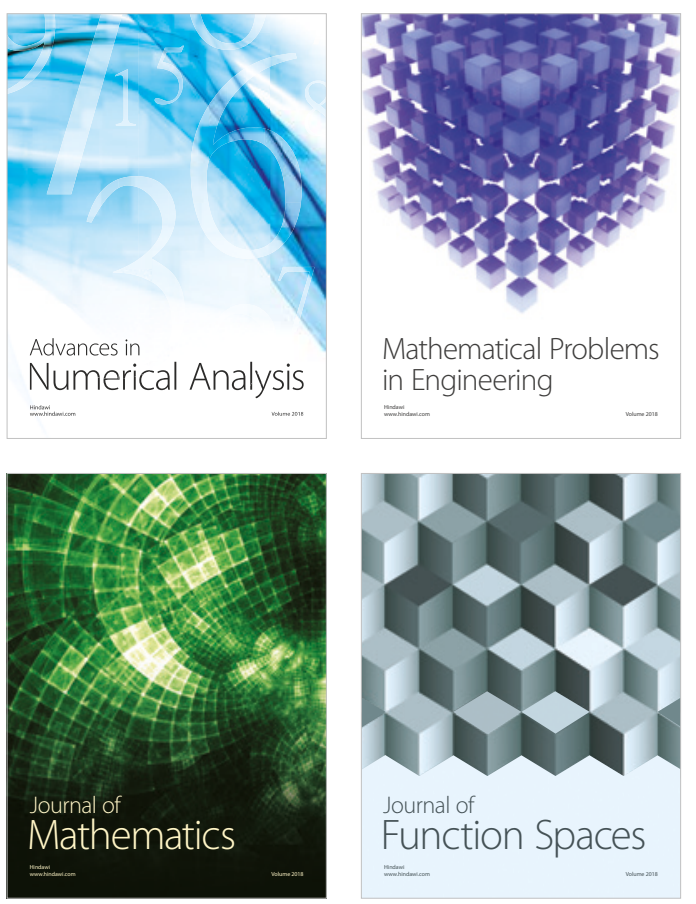

Mathematical Problems in Engineering

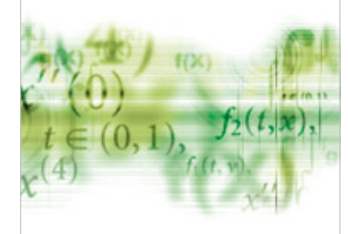

International Journal of

Differential Equations

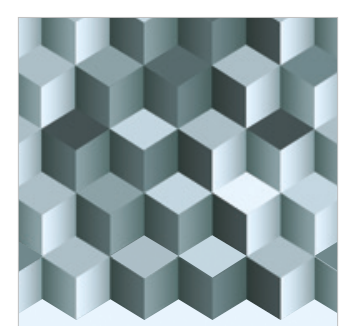

Journal of

Function Spaces
The Scientific

World Journal

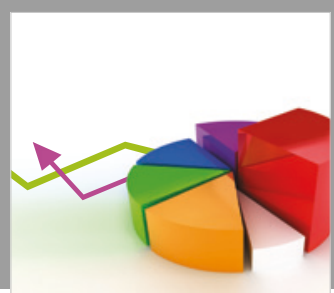

Journal of

Probability and Statistics
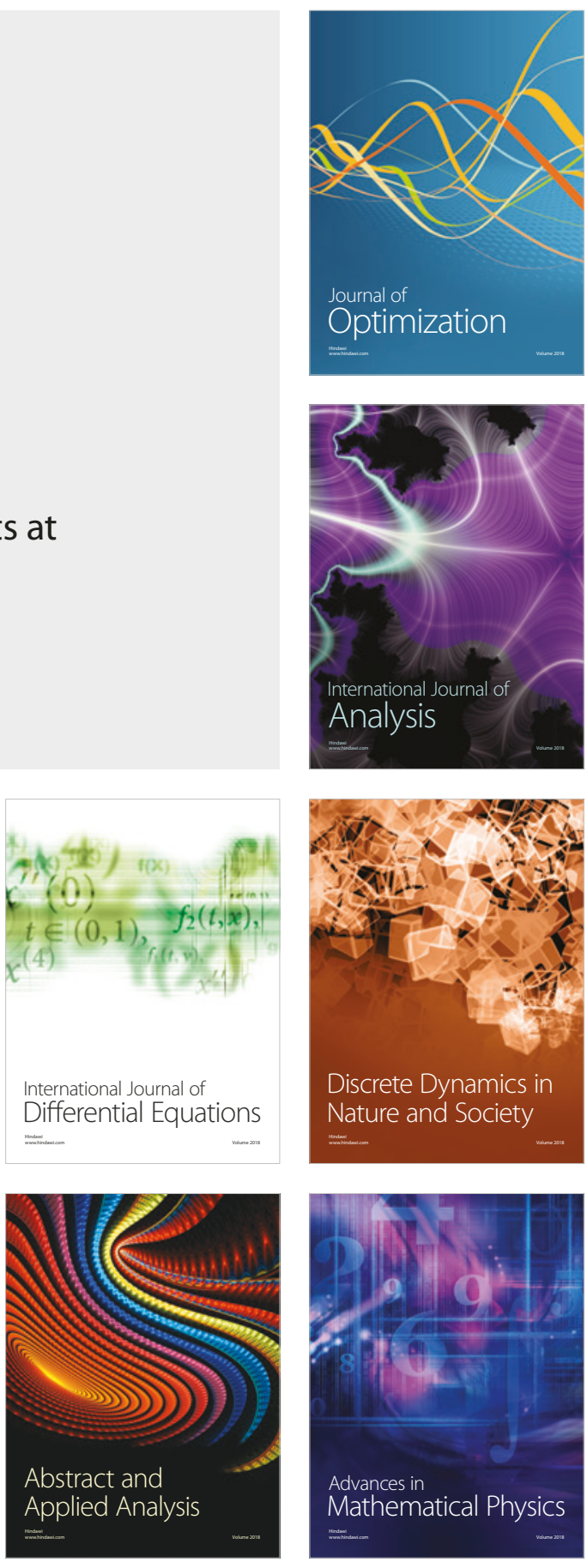